\title{
An Innovative Approach to Health Care Delivery for Patients with Chronic Conditions
}

\author{
Janice L. Clarke, RN, ${ }^{1}$ Scott Bourn, PhD, RN, EMT-P, ${ }^{2}$ Alexis Skoufalos, EdD, \\ Eric H. Beck, DO, MPH, NREMT-P, ${ }^{2}$ and Daniel J. Castillo, MD, MBA ${ }^{2}$
}

\begin{abstract}
Although the health care reform movement has brought about positive changes, lingering inefficiencies and communication gaps continue to hamper system-wide progress toward achieving the overarching goal—higher quality health care and improved population health outcomes at a lower cost. The multiple interrelated barriers to improvement are most evident in care for the population of patients with multiple chronic conditions. During transitions of care, the lack of integration among various silos and inadequate communication among providers cause delays in delivering appropriate health care services to these vulnerable patients and their caregivers, diminishing positive health outcomes and driving costs ever higher. Long-entrenched acute care-focused treatment and reimbursement paradigms hamper more effective deployment of existing resources to improve the ongoing care of these patients. New models for care coordination during transitions, longitudinal high-risk care management, and unplanned acute episodic care have been conceived and piloted with promising results. Utilizing existing resources, Mobile Integrated Healthcare is an emerging model focused on closing these care gaps by means of a round-the-clock, technologically sophisticated, physician-led interprofessional team to manage care transitions and chronic care services on-site in patients' homes or workplaces.
\end{abstract}

\section{Introduction}

$\mathbf{S}^{\mathrm{n}}$ NCE ITS IMPLEMENTATION in 2010, the Patient Protection and Affordable Care Act (ACA) has succeeded in providing more people with access to care and improving value on a number of fronts (eg, banning preexisting conditions as a reason to deny health insurance coverage, instituting exchanges that enable consumers to comparison shop for health insurance plans, allowing adult children up to age 26 coverage under their parents' health insurance). However, the complexity of the large, fragmented, and uncoordinated US health care system continues to prove challenging, despite ongoing efforts to address escalating health care costs and suboptimal quality outcomes.

\section{The problem}

Persistent inefficiencies, resistance to change, and multiple interrelated issues across the system prevent critical information and resources from reaching providers and patients in a timely manner. Strategic vision, coupled with the ability to mobilize and deliver appropriate resources to patients in the community, is needed so that health care professionals can provide accessible, safe, well-coordinated, cost-effective, high-quality care.

Access to healthcare services. Continued barriers to access range from lack of availability to high prices to lack of insurance coverage. In the nation's 4 largest states, $12 \%-$ $30 \%$ of residents lacked health insurance coverage or experienced problems getting needed care in $2014 .{ }^{1}$ In many rural areas, an inadequate supply of health care professionals restricts access to needed services, challenging the health care system to utilize and deploy clinical and material resources in different ways. ${ }^{2}$ A major weakness in typical outpatient and inpatient care delivery systems is that primary care professionals, paramedics, emergency physicians, and hospitalists function in unintegrated silos that impede

\footnotetext{
${ }^{1}$ Jefferson College of Population Health, Philadelphia, Pennsylvania.

${ }^{2}$ Evolution Health, Dallas, Texas.
}

(c) Clarke et al. 2016; Published by Mary Ann Liebert, Inc. This Open Access article is distributed under the terms of the Creative Commons Attribution Noncommercial License (http://creativecommons.org/licenses/by-nc/4.0/) which permits any noncommercial use, distribution, and reproduction in any medium, provided the original author(s) and the source are credited. 
care coordination, inhibit communication, compromise quality, and raise costs. These and other modifiable barriers to access lead invariably to unmet health needs, delays in receiving appropriate care, and preventable hospitalizations.

Cost of care issues. Although the rate of escalation has diminished recently, health care costs remain high. In a recent Gallup survey, $42 \%$ of Americans named either the cost of health care or access to health care as the top US health issues. ${ }^{3}$ According to one recent projection, the rate of increase in the cost of employer-sponsored health care benefits in 2015 will equal about $4.1 \%{ }^{4}$ Equally troubling are estimates that up to one third of the more than $\$ 2.8$ trillion spent on health in the United States each year may be classified as waste-waste that is related largely to failures of care delivery, care coordination, and overuse.

Health care quality and safety issues. Serious gaps in care guidelines and processes continue to have an adverse impact on the quality and safety of care, particularly in outpatient settings. ${ }^{5}$ Specifically, poor care coordination processes lead to failures in transmitting critical patient information, adverse drug interactions, conflicting treatment plans, and/or lapses in necessary treatment. Failures in patient communication and patient education were among the root causes of 197 sentinel events (eg, suicide, falls, wrongside surgery) compiled by The Joint Commission from January 2014 to October $2015{ }^{6}$

Health information technology (HIT) issues. Although health care communities across the country have made some strides in adopting, implementing, and using HIT to share relevant patient information, the lack of widespread adoption of a reliable system that can share and integrate communication across institutional and organizational boundaries is a significant hindrance to efforts to improve care coordination. ${ }^{7}$

Chief among these issues that hamper progress are persistent inefficiencies and wasted resources that compromise access, health outcomes, and value. These interrelated problems occur with alarming regularity:

- during transitions of care,

- in the longitudinal management of medically complex patients, and

- when individuals with chronic disease have clinical episodes that require acute intervention.

Clearly, there is a critical need for innovative solutions that improve care coordination and communication among clinicians, patients, and their families/caregivers during these high-risk intervals, facilitating medically appropriate just-in-time care delivery by interprofessional teams.

\section{Pivotal Role of Coordinated Care Transitions}

The term transitions of care refers to the movement of patients-between health care locations, providers, or levels of care as their conditions and care needs change — and the set of actions designed to ensure coordination and continuity. Effective transitional care can prevent medical errors, identify issues for early intervention, avert unnecessary hospitalizations and readmissions, support consumers' preferences and choices, and avoid duplication of services, thereby improving the quality of care while utilizing resources more effectively. Ideally, care transition activities are based on a comprehensive care plan and the availability of well-trained practitioners who have current information about the patient's treatment goals, preferences, and health or clinical status.

In reality, a large and growing evidence base raises serious concerns for patients undergoing transitions across care settings, especially those with continuous complex needs, who are particularly vulnerable to experiencing the poor-quality outcomes associated with failures in provider communication and fragmentation in care. ${ }^{9}$ A 2011 report revealed that poorly coordinated transitions from the hospital to other care settings cost an estimated $\$ 12$ billion to $\$ 44$ billion per year ${ }^{10}$ and often result in adverse health outcomes (eg, injuries related to medication errors, postmedical procedure complications, infections, falls). ${ }^{11}$

In 2012, under the terms of the ACA, the Centers for Medicare \& Medicaid Services (CMS) launched an initiative to improve care transitions, offering technical assistance, tools, and other resources for states and their providers and instituting penalties for hospitals with high rates of hospital readmission for 3 conditions: myocardial infarction, heart failure, and pneumonia. ${ }^{12}$ Created under Section 3026 of the ACA, the CMS Community-based Care Transitions Program has 72 test sites across the country. Although hospitals faced reimbursement reductions and new value-based payment models (eg, bundled payments) ${ }^{12}$ under the law, they also became eligible for incentives by effectively coordinating transitions, providing appropriate levels of care, and ensuring safe transitions. ${ }^{12}$

\section{Research and results}

A variety of interventions have been tested to improve transitions of care, including the Care Transitions Intervention Model, ${ }^{9}$ Project Better Outcomes for Older adults through Safe Transitions (Project BOOST), ${ }^{13}$ and the Transitional Care Model. ${ }^{14}$ In well-constructed studies, these programs demonstrated reduced rehospitalization rates at 30, 60, and 90 days; lengthening the interval between hospitalizations; lower hospital costs; and a reduction in total health care costs. While each of the interventions described was unique, they shared a number of successful strategies:

- Transitional care coordination utilizing a transitional coach or coordinator (a nurse, social worker, community health worker, transitions coach, or other health professional) who performs a comprehensive patient/ family assessment before discharge, leads the development of a discharge plan, delivers patient and family education, and helps patients to take a more active and informed role in their transition.

- Enhanced communication between acute care and postacute care providers to assure critical information follows the patient through the transition (eg, facilitation of a discharge conference between acute and post-acute teams to assure that discharge records are complete and received by post-acute care providers).

- Increased patient and family engagement and participation in the discharge plan. 
- Support in execution of the postdischarge plan, including assistance with making follow-up appointments, home visits to assess the home environment and provide feedback to family and caregivers on their delivery of care, and care coordination between multiple providers.

\section{Longitudinal High-Risk Care}

The size of the chronic disease population — particularly patients with multiple comorbidities that require more health care resources-is projected to grow, reaching 157 million Americans by $2020 .{ }^{15}$ Various provisions of the ACA encourage chronic disease management (DM) by incentivizing self-management by patients, offering some reimbursement opportunities for providers of chronic DM services, and/or providing government support for the development of chronic DM programs, but substantial barriers to optimal care remain. ${ }^{16}$

There are significant differences between acute and chronic disease that require different approaches to care. The American health care system is built on an acute care paradigm; in general, acute care problems have a rapid onset, are short in duration, and result from a single cause. Chronic care problems are slower to develop, longer in duration, and have multiple causes, some of which occur years before the onset of symptoms. These differences limit the current system's ability to deal effectively with a number of unique challenges in managing chronic disease:

- The social, behavioral, and psychosocial elements associated with chronic disease (eg, the often unrecognized elements of self-image related to being a person with disease).

- The need for continuing care, often throughout the remainder of the patient's life.

- The influence of chronic disease on the patient's extended family and the very real need for the family's ongoing support for long-term success.

- The influence of lifestyle factors in both the causation and long-term management of chronic disease.

Caring for the population of patients with chronic conditions requires a new paradigm-one that encompasses longitudinal care and unplanned episodes of care. To a large extent, the development and implementation of such a system will hinge on addressing 4 specific challenges ${ }^{17}$ :

1. Realigned Reimbursement-In general, payment for health care services is triggered by acute care episodes. There must be a mechanism whereby providers are compensated to manage a broad range of chronic conditions that never resolve and that are not characterized by episodes of care.

2. Team-Based Care-An adequate number of nonphysician health care team members in disciplines such as nursing, social work, community health coaching, and pharmacy must be trained and available to coordinate proven team-based care.

3. Patient and Family Engagement-Expanded opportunities for patient and family engagement in selfmanagement programs are essential for improving patients' ability to manage their conditions and adhere to treatment plans.
4. Information Sharing-The current acute episodefocused medical record system must be redesigned to improve clinicians' ability to share information regarding patients with chronic disease and facilitate the use of evidence-based decision support in their care.

\section{Research and results}

A number of strategies have demonstrated an ability to improve health status and reduce utilization of health care resources for the population of patients with chronic conditions; for instance-

DM programs were pioneered in the 1990s during the managed care era and have since been widely adopted by health care delivery systems and public and private payers. DM programs are designed to improve the health of persons with specific chronic conditions, thereby reducing health care service use and the costs associated with avoidable complications (eg, emergency department [ED] use, hospitalizations). The typical DM program features a number of important elements:

- Robust population identification processes used to target individuals with single or multiple costly chronic conditions.

- Evidence-based clinical practice guidelines to ensure consistency in treatment across the targeted population and for use by providers to educate patients.

- Collaborative practice models (eg, interprofessional practice, team-based care) to help patients manage their conditions.

- Patient self-management education, including additional support for adhering to medication regimes, counseling, home visits, 24-hour call centers, and appointment reminder systems.

- Methods for measuring both process and clinical outcomes, including patient satisfaction in addition to health care service use and expenditures.

- Routine communication among treatment teams, patients, and program sponsors to track progress in condition management.

Common among both government programs and private health plans for 2 decades, DM programs have been shown to reduce costs, ${ }^{18}$ generate modest improvements in quality of life, ${ }^{19}$ and elevate the level of patient satisfaction. ${ }^{20}$

Developed by researchers at Johns Hopkins University, the Guided Care model assists primary care practices in meeting the complex needs of patients with multiple chronic conditions (MCCs) by means of a Guided Care nurse who works closely with patients, physicians, and others to provide coordinated patient-centered care. ${ }^{21}$ Studies have indicated that Guided Care

- improved patients' perception of the quality of their care, ${ }^{22}$

- improved family and caregivers' perceptions of care quality, ${ }^{23}$

- improved physicians' satisfaction with chronic care, ${ }^{24}$

- produced high job satisfaction among nurses, ${ }^{25}$

- helped reduce the use of expensive services (eg, 29\% fewer home health visits, $13 \%$ fewer hospitalizations), ${ }^{26}$ and 
- reduced the use of services in an Integrated Delivery System (eg, 47\% fewer skilled nursing facility admissions, $17 \%$ fewer ED admissions). ${ }^{25}$

\section{Unplanned Episodic Care}

Generally, patients with comorbid physical and mental health problems require more unscheduled episodic care, especially if they are elderly, increasing unplanned utilization of health care resources and their associated costs. Data from a 2012 CMS report show that Medicare beneficiaries with MCCs were significantly more likely to have multiple hospital admissions and readmissions, ED visits, and home health visits than a comparable population without 0-1 chronic conditions. ${ }^{26}$ Low levels of health literacy and patient activation also have been documented in this patient population, further increasing the potential for acute care service utilization. ${ }^{27}$

A unique subset of this population, sometimes referred to as super-utilizers, visits the ED 10 or more times each year, with an associated high frequency of hospital admissions. ${ }^{28,29}$ A longitudinal analysis of 4774 publicly insured or uninsured patients found that the $3 \%$ of adult patients who met superutilizer criteria accounted for $30 \%$ of adult care costs. ${ }^{29}$

The Clinically Appropriate and Cost-Effective Placement Project undertaken by the Alliance for Home Health Quality determined that Medicare beneficiaries with chronic conditions are more likely to be readmitted to inpatient care settings within 60 days of discharge. Of all post-acute care readmissions, $60 \%$ of episodes are readmitted directly from the community. Further analysis suggested that there may be great potential for strategies that provide ongoing, clinically appropriate chronic care management to reduce the frequency of avoidable admissions and readmissions across all episode types. ${ }^{30}$

Unplanned utilization of health care resources has substantial cost implications; for instance, the 3 conditions with the greatest number of 30-day all-cause readmissions for Medicare patients resulted in $\sim \$ 4.3$ billion in hospital costs. ${ }^{31,32}$

\section{Research and results}

A systematic review of 35 studies examining the relationship between MCCs and health care utilization outcomes (ie, physician, hospital, and medication use) and costs outcomes (ie, medication costs, out-of-pocket costs, total health care costs) for elderly general populations revealed a positive association of MCCs and significantly increased use/costs with each incremental condition. ${ }^{27}$ Several of the studies indicated a near exponential relationship between MCCs and costs. The rising prevalence of MCCs, their substantial costs, and concerns that current care approaches may be inappropriate for many such patients raise important questions for providers, payers, and policy makers alike. ${ }^{27}$

Another recent study comparing the characteristics of US adults by the frequency of ED utilization-specifically the prevalence of chronic diseases, outpatient primary care, and mental health utilization-concluded that frequent ED users had a large burden of chronic diseases (eg, coronary artery disease, stroke, asthma) that also required a high number of outpatient resources. The authors concluded that interventions designed to divert frequent ED use should focus on chronic DM and access to outpatient services, particularly for Medicaid beneficiaries and other high-risk subpopulations. ${ }^{28}$

Evidence-based strategies have demonstrated an ability to reduce the frequency of unplanned episodes of care and to decrease preventable ED use and inpatient hospitalization when they occur. For instance, North Carolina instituted a statewide, population-based transitional care initiative to prevent recurrent hospitalization among high-risk Medicaid recipients with complex chronic medical conditions. A study of patients hospitalized during 2010-2011 found that those who received transitional care were $20 \%$ less likely to experience readmission during the subsequent year compared with clinically similar patients who received usual care. The authors concluded that such locally embedded, targeted care coordination interventions can effectively reduce hospitalizations for high-risk populations. ${ }^{33}$

Although the existing health care delivery system addresses some of the aforementioned elements, serious gaps in care remain. In particular, uncoordinated discharge processes and shortcomings in the availability of and access to home care and visiting nurse services continue to impede improvement efforts. In some instances, existing reimbursement policies preclude the management of episodes in the most appropriate setting. One study revealed that among Medicare beneficiaries who placed 911 calls to request an ambulance, $\sim 34.5 \%$ had a low acuity diagnosis that might have been managed outside the ED. If Medicare had the flexibility to reimburse emergency medical services (EMS) to provide necessary medical management for select 911 calls, the authors estimated that the federal government could save \$283-\$560 million or more per year while improving continuity of patient care. ${ }^{34}$

\section{Mobile Integrated Healthcare: An Emerging Model Focused on Closing Gaps and Improving Health Outcomes}

The preceding discussion telegraphs certain elements that are essential to an effective solution (ie, improving care transitions, managing longitudinal high-risk patients, and reducing unplanned episodes of care require an interdisciplinary team that specializes in transition management, is available and deployable on demand, and is in constant contact with all stakeholders). The capacity to coordinate care of multiple patients with unique problems who receive regular care from diverse and disconnected providers-and to do so on demand at the level required-is challenging. However, most communities have an EMS system, an often untapped resource that regularly performs comparable tasks as follows:

- Receiving incoming requests for emergency services through the 911 system and requests for nonemergent medical transportation through the phone or online ordering services.

- Using validated, physician-developed evidence-based criteria to prioritize requests to ensure that resources are dispatched according to the degree of urgency within minutes for emergent and urgent needs in most instances.

- Matching resources to patient needs.

- Deploying resources geographically to reduce transit time and assure prompt and efficient response. 
- Ensuring system status and staffing to support appropriate and efficient utilization.

- Tracking resources in real time, 24/7, to assure geographic availability of resources and maintain safety of responding professionals.

A community EMS system's ability to manage, prioritize, schedule, and respond to clinical requests, as well as track community health resources, makes it an ideal partner for pioneering new approaches to caring for chronically ill patients and tending to nonurgent events outside of hospital EDs. Historically EMS and public safety have not been meaningfully linked to post-acute and transitional care initiatives.

The concept of mobile integrated healthcare (MIH) and community paramedicine has emerged as a promising framework for more complex and comprehensive medical care delivery. Using a broad variety of resources and clinical professionals-including community health workers, traditional emergency medicine personnel, as well as nurses, social workers, pharmacists, advanced practice providers, and physicians - these programs address wellness, prevention, care for chronically ill patients, posthospital discharge care, social support networks, and patient education/engagement for a locally defined population. ${ }^{35}$

MIH is an innovative approach aimed at closing these gaps by utilizing the core competencies of a professional staff of clinical specialists who are already available within a community providing community health, post-acute care, and EMS. Originally designed as a paramedicine program to address health care needs in rural areas where there are fewer physicians and residents tend to display poorer health outcomes (eg, higher rates of substance use, infant and adolescent mortality, self-reported obesity), an expanded MIH concept has been used in nonrural areas since $2012 .{ }^{36}$ In addition to broadening the provider scope of practice to cover chronic disease surveillance, community health education, and prevention, the new MIH model expanded the role of EMS providers and incorporated primary care teams, hospice and palliative care providers, and other home care and community-based service organizations. Already accustomed to working in and with the communities it serves, MIH provides comprehensive, physician-led, patient-centered population management solutions across the care continuum, including mobile integrated comprehensive care coordination, telehealth and telemedicine, in-home and mobile care, and longitudinal risk management. Importantly, the model is locally adaptable for both disease-specific interventions and more broadly defined patient populations.

Community-based and technologically sophisticated, the $\mathrm{MIH}$ model focuses on delivering necessary services at the most appropriate level of care and specializes in the care and management of complex patient populations at home and in other community-based settings. It accomplishes this by means of integrating clinical, logistical, analytical, and educational competencies in a collaborative effort that provides patient-centered, team-based population-oriented care.

\section{Central elements of an MIH model}

A 24/7/365 interprofessional approach. A physician-led interprofessional care team is tailored to a defined population and individual patient needs within that population. The team may draw on pre-acute, acute, and post-acute care expertise of an emergency, hospitalist, primary care, or behavioral health provider, a clinical specialist trained in transitional care, a pharmacist, a mobile, telehealth, or inhome team member. The interprofessional approach facilitates safer, higher quality, and more cost-effective care (ie, needs-matched care by the most appropriate provider in the most appropriate setting).

Command center (CC). The aforementioned resources can provide value to patients and the community only if incoming requests for service are received, accurately matched to appropriate resources, and tracked on a 24-hour basis. A core competency of public safety and EMS systems within communities is management and/or coordination of an operational dispatch and communications center that performs the following:

- Receives incoming 911 and nonemergency requests for medical and social services.

- Matches or navigates requests to available, proximate, and/or appropriate resources.

- Actively tracks and manages resources throughout the system to best optimize delivery.

In most successful MIH programs, this capability is expanded to include management of incoming requests for MIH services such as home visits, hospice services, medication delivery, and/or referral of requests to the most appropriate local organization. The MIH program's ability to coordinate requests for service and to match and track resources is critical to achieving coordination of services.

Transitional care. Like hospitalist, the relatively new term transitionalist is still unfamiliar, even for many in the health care field. A transitionalist team focuses on improving care transitions by assessing, managing, and providing support for clinical and psychosocial risk factors. Transitionalist teams excel in needs matching, patient education, information sharing, and handoff communication with other stakeholders in care. ${ }^{37}$

The well-documented Care Transitions Intervention ${ }^{21}$ and Transitional Care Model $^{14}$ use different approaches, but both provide patients with tools and the necessary support to manage their own conditions. ${ }^{38}$ Coordinated by the CC, multiprofessional transitionalist teams are critical to the successful transition of patients and the associated reduction in complications and readmissions.

Longitudinal high-risk care. Wide variability in the quality of care for the high-risk patient population accounts for the largest percentage of health care costs. To effectively address this issue, a physician or other member of the interprofessional team conducts in-home/at-work visits for vulnerable patients, providing comanagement on behalf of/in communication with primary care providers, health plan care managers, and/or specialists for medically complex, highest risk, highest cost, and highest touch patients.

Longitudinal high-risk interventions target self-management of chronic diseases with the goal of minimizing exacerbations, reducing hospital (re)admissions, and decreasing preventable ED and 911 call utilization. MIH programs close unaddressed gaps (eg, access to transportation, declining functional status, 
community support, safe independence at home), thereby avoiding diversions that delay necessary care and improving quality and cost outcomes. Tangentially, these interventions ease the demand on first responders by reducing nonemergent calls.

Advanced illness management. Respectful coaching and care are provided for patients with advanced chronic illnesses and functional decline (eg, advanced-stage congestive heart failure) with a focus on managing symptoms and medication, providing comfort, coordinating care, planning for the future, and improving quality of life. This holistic approach includes the patient's family and caregivers as goals of care change from curative to palliative in orientation.

Unplanned acute care. Even fully engaged patients with well-managed chronic diseases occasionally need additional support; thus, effective population health management must encompass both planned and unplanned care. The CC coordinates care for unanticipated needs using mobile clinicians and telemedicine capabilities with all services delivered by physicians in collaboration with other members of the MIH team.

\section{Discussion}

New models for transitions of care, longitudinal care, and unplanned episodes of care have proven effective in coordinating care between settings, improving the quality and efficiency of care, and reducing the overall cost of care. The $\mathrm{MIH}$ model described herein builds on these models in several ways.

Working in conjunction with rather than competing with existing community health care services, MIH uses previously untapped capacity of medical transport services to avert nonacute ED visits. Analyses reveal that of 136.3 million ED visits, only $11.9 \%$ result in a hospital admission. $^{39}$ These unnecessary ED visits cost the US health care system more than $\$ 4.4$ billion annually. Studies vary, but inappropriate ED visits account for $8 \%^{39}-27 \%{ }^{40}$ of total visits. Few dispute that more appropriate, less expensive care could be received in an alternate setting (eg, the patient's home). By mobilizing all existing resources more efficiently and effectively_including mobile health services-the MIH model enables active patient management in the most appropriate setting (eg, many patients with chronic conditions do not require ED-level care when their conditions begin to exacerbate).

The MIH model dovetails with the ongoing transformation in health care delivery brought about by the implementation of the ACA-from acute/inpatient centered to managed/outpatient centered. The new care paradigm is one in which care is delivered directly to patients in their homes and/or work environments, and clinical and social interventions are provided before conditions become acute. This translates into a clinically appropriate reduction in ED and hospital utilization-a distinct advantage for providers wishing to avoid financial penalties for inappropriate level of care and readmission.

\section{Population health implications}

Delivering appropriate care that is high quality and costeffective is an imperative. For vulnerable populations (eg, chronic and complex conditions, frail and elderly, Medicaid, behavioral health conditions), it presents a substantial challenge. A major weakness in the typical care delivery system is that emergency physicians, hospitalists, paramedics, primary care physicians, and home care and social service providers function independently in unintegrated silos that are associated with fragmentation, lack of coordination, variability in quality and health outcomes, and unnecessary costs. An overarching goal of health care reform is to dismantle the silos-more precisely, to evolve from a fragmented system to an integrated model that shares crucial information and provides services more efficiently at a lower cost. Emerging MIH models seek to change this dynamic, capitalizing on improved synergy to improve the quality and safety of care, reduce waste and inefficiency, and realize cost savings.

The term clinical integration is generally described as the extent to which patient care services are coordinated across the spectrum of functions, activities, and sites over time to maximize the value of services delivered to a defined population. Most health care experts now agree that breaking down the clinical silos of complex health care organizations (ie, integration) is beneficial. MIH is one of a growing number of integrated models that are proving the hypothesis.

\section{Conclusion}

Assuring access to high-quality, safe, integrated, and wellcoordinated care is tantamount to improving population health outcomes, reducing wasteful spending, and reversing the spiraling cost curve. Unfortunately, a multitude of interrelated problems continue to impede progress toward these goals, and these considerable issues are most evident in caring for the growing population of patients with multiple, complex chronic conditions. MIH is an emerging model that leverages EMS systems to effectively address the key issues of care transitions, longitudinal care, and unplanned episodes of care by using existing resources more efficiently and enabling data and information sharing among health systems and other providers. Readily adaptable to meet the health needs of populations in any locality, the model is designed to deliver chronic and urgent care whenever and wherever needed.

\section{Acknowledgment}

The authors are grateful for the guidance from William K. Atkinson, PhD, MPH, MPA, EMT-P.

\section{Author Disclosure Statement}

The authors declared the following potential conflicts of interest with respect to the research, authorship, and/or publication of this article; Ms. Clarke and Dr. Skoufalos are employed by the Jefferson College of Population Health. Drs. Bourn, Beck, and Castillo are employed by Evolution Health, a provider of Mobile Integrated Health services. The authors received the following financial support for this article: The Jefferson College of Population Health received compensation from Evolution Health for manuscript preparation and editorial services.

\section{References}

1. The Commonwealth Fund. Health Care Coverage and Access in the Nation's Four Largest States: Results from the 
Commonwealth Fund Biennial Health Insurance Survey, 2014. 2015. www.commonwealthfund.org/publications/issuebriefs/2015/apr/coverage-and-access Accessed April 20, 2016.

2. US Department of Health and Human Services, Health Resources and Services Administration. Shortage Areas, Health Professional Shortage area (HPSA)-Basic Primary Medical Care. http://datawarehouse.hrsa.gov/Tools/HDW Reports/Reports.aspx Accessed December 30, 2015.

3. Riffkin R. Americans Again Cite Cost and Access as Top Health Issues. November 18, 2015. www.gallup.com/poll/ 186722/americans-again-cite-cost-access-top-health-issues .aspx Accessed December 30, 2015.

4. Willis Towers Watson. US Employers Expect Rate of Increase in Health Care Costs in 2015 to Remain Low But Well Above Inflation. October 6, 2015. https://towerswatson .com/en-US/Press/2015/10/rate-of-increase-in-health-carecosts-in-2015 Accessed April 20, 2016.

5. Singh H, Meyer AND, Thomas EJ. The frequency of diagnostic errors in outpatient care: estimations from three large observational studies involving U.S. adult populations. BMJ Qual Saf. 2014;23:727-731.

6. The Joint Commission. Transitions of Care: engaging Patients and Their Families. November 2015. www.jointcommi ssion.org/assets/1/23/Quick_Safety_Issue_18_November_ 20151.PDF Accessed April 20, 2016.

7. Agency for Healthcare Research and Quality. Care Coordination, Quality Improvement. October 2014. www.ahrq. gov/research/findings/evidence-based-reports/caregaptp.html Accessed January 4, 2016.

8. National Transitions of Care Coalition. The National Transitions of Care Coalition. www.ntocc.org/aboutus.aspx Accessed April 20, 2016.

9. Johns Hopkins University. Guided Care: comprehensive Primary Care for Complex Patients. 2013. http://guidedcare. org/program-history-results.asp Accessed October 28, 2015.

10. Hansen LO, Young RS, Hinami K, Leung A, Williams MV. Interventions to reduce 30-day rehospitalization: a systematic review. Ann Intern Med 2011;155:520-528.

11. Kim CS, Flanders SA. Transitions of care. Ann Intern Med 2013;58(5 pt 1): ITC3-ITC1.

12. Burton R. Health Policy Brief: Improving Care Transitions. September 13, 2012. www.healthaffairs.org/healthpolicy briefs/brief.php?brief_id=76 Accessed October 28, 2015.

13. Society of Hospital Medicine. Project Better Outcomes for Older Adults Through Safe Transitions (Project BOOST). www.hospitalmedicine.org/Web/Web/Quality__Innovation/ Mentored_Implementation/Project_BOOST/Project_BOOST .aspx Accessed April 25, 2016.

14. Naylor MD. Transitional care for older adults: a costeffective model. LDI Issue Brief 2004;9:1-4.

15. Priester R, Kane RL, Totten AM. How the current system fails people with chronic illnesses. Society of Actuaries. 2005. file:///C:/Users/jlc114/Downloads/M-HB05-1_VI.pdf. Accessed April 25, 2016.

16. Cartwright-Smith L. Chronic Disease Management. February 23,2011 . www.healthreformgps.org/resources/chronicdisease-management/ Accessed April 25, 2016.

17. Harris MF, Zwar NA. Care of patients with chronic disease: the challenge for general practice. Med J Aust 2007;187: 104-107.

18. Rubin R, Deitrich KA, Hawk AD. Clinical and economic impact of implementing a comprehensive diabetes disease management program in managed care. J Clin Endocrinol Metab 1998;83:2635-2642.
19. NGA Center for Best Practices. Disease management: the new tool for cost containment and quality care. Issue Brief. Washington, DC: National Governors Association, 2003.

20. Rich M, Beckham V, Wittenberg C, Leven CL, Freedland $\mathrm{KE}$, Carney RM. A multidisciplinary intervention to prevent the readmission of elderly patients with congestive heart failure. N Engl J Med 1995;333:1190-1195.

21. Coleman EA, Parry C, Chalmers S, Min S. The care transitions intervention: results of a randomized controlled trial. Arch Intern Med 2006;166:1822-1828.

22. Boult C, Leff B, Boyd CM, et al. A matched-pair clusterrandomized trial of guided care for high-risk older patients. J Gen Intern Med 2013;28:612-621.

23. Wolff JL, Giovannetti ER, Boyd CM, et al. Effects of guided care on family caregivers. Gerontologist 2010;50: 459-470.

24. Marsteller JA, Hsu YJ, Reider L, et al. Physician satisfaction with chronic care processes: a cluster-randomized trial of guided care. Ann Fam Med 2010;8:308-315.

25. Boult C, Reider L, Frey K, et al. Early effects of "guided care" on the quality of health care for multimorbid older persons: a cluster-randomized controlled trial. J Gerontol A Biol Sci Med Sci 2008;63:321-327.

26. Centers for Medicare \& Medicaid Services. Chronic Conditions Among Medicare Beneficiaries. Chartbook: 2012 edition. www.cms.gov/research-statistics-data-and-systems/ statistics-trends-and-reports/chronic-conditions/downloads/ 2012chartbook.pdf Accessed April 25, 2016.

27. Lehnert T, Heider D, Leicht H, et al. Review: health care utilization and costs of elderly persons with multiple chronic conditions. Med Care Res Rev 2011;68:387-420.

28. Vinton DT, Capp R, Rooks SP, Abbott JT, Ginde AA. Frequent users of US emergency departments: characteristics and opportunities for intervention. Emerg Med J 2014;31:526-532.

29. Johnson TL, Rhinehart DJ, Durfee J, et al. For many patients who use large amounts of health care services, the need is intense yet temporary. Health Aff 2015;34:13121319.

30. Alliance for Home Health Quality and Innovation. Hospital readmission and admissions-Executive summary: CACEP Working Paper \#4. www.ahhqi.org/images/pdf/cacep-wp4executive-summary.pdf Accessed April 25, 2016.

31. Begum N, Donald M, Ozolins IZ, Dower J. Hospital admissions, emergency department utilisation and patient activation for self-management among people with diabetes. Diabetes Res Clin Pract 2011;93:260-267.

32. Hines AL, Barrett ML, Jiang HJ, Steiner CA. Conditions with the largest number of adult hospital readmissions by payer, 2011. Healthcare Cost and Utilization Project Statistical Brief \#172. Rockville, MD: Agency for Healthcare Research and Quality, 2014.

33. Jackson CT, Trygstad TK, DeWalt AD, DuBard CA. Transitional care cut hospital readmissions for North Carolina Medicaid patients with complex chronic conditions. Health Aff 2013;32:1407-1415.

34. Alpert A, Morganti KG, Margolis GS, Wasserman J, Kellermann AL. Giving EMS flexibility in transporting lowacuity patients could generate substantial Medicare savings. Health Aff 2013;32:2142-2148.

35. Choi BY, Blumberg C, Williams K. Mobile integrated health care and community paramedicine: an emerging emergency medical services concept. Ann Emerg Med 2015;67:361-366. 
36. Beck E, Craig A, Beeson J. Mobile integrated healthcare practice: a healthcare delivery strategy to improve access, outcomes, and value. 2013. www.acep.org/uploadedFiles/ ACEP/Practice_Resources/disater_and_EMS/MIHP_white paper\%20FINAL1.pdf Accessed May 25, 2016.

37. Ota KS, Beutler DS, Gerkin RD, Weiss JL, Loli AI. Physician-directed heart failure transitional care program: a retrospective case study. J Clin Med Res 2013;5:335-342.

38. California Healthcare Foundation. Navigating care transitions in California: two models for change. www.healthreform.ct .gov/ohri/lib/ohri/1._CA_TWO_MODELS.pdf Accessed August 31, 2015.

39. Centers for Disease Control and Prevention. National Center for Health Statistics. Emergency Department Sta- tistics. www.cdc.gov/nchs/fastats/emergency-department .htm Accessed May 2, 2016.

40. Weinick RM, Burns RM, Mehrotra A. Many emergency department visits could be managed at urgent care centers and retail clinics. Health Aff 2010;29:1630-1636.

Address correspondence to: Janice L. Clarke, RN Jefferson College of Population Health 901 Walnut Street, 10th Floor Philadelphia, PA 19107

E-mail: janice.clarke@jefferson.edu 